\title{
«Work and Care» - concilier profession, vie privée et soins à des proches
}

\section{Ursula Steiner-König}

Déléguée de la FMH au comité central de la Journée des malades
Aujourd'hui, penser que des enfants, adultes et en pleine vie professionnelle, prendront en charge leurs parents appartient à une époque révolue. Notre société vieillit, et irrémédiablement, nous pouvons en arriver au point de ne plus être en mesure de tout assumer seuls. Mais sur qui pouvons-nous compter, qui viendra nous apporter son concours? Le comité central de la Journée des malades s'est emparé du sujet pour sensibiliser l'opinion sur la question. En effet, les problèmes qui en découlent sont de plus en plus nombreux et nous concernent tous.

Une approche traditionnelle voudrait que les enfants des personnes nécessitant des soins se dévouent pour s'occuper d'elles et les prendre en tâches? Combien de temps faudra-t-il pour accomplir tout ce qui doit être fait? Est-ce que je me sens émotionnellement prêt-e à assumer cette tâche pour mes parents? Quel respect suis-je en droit d'attendre de leur part? Faut-il que je revoie mes attentes? Comment réagira mon entourage actuel au fait qu'une partie de mon énergie, de mon attention et de mon temps soit également consacrée à d'autres personnes? Combien de temps risque de durer cette nouvelle situation? Quelles possibilités de remplacement existent pour parer les pics? Devrais-je réduire, repenser ou repousser mes ambitions professionnelles? Est-ce tout simplement envisageable? Qu'en est-il des vacances et du temps libre? Financière-

\section{Concilier profession, vie privée et soins à des proches n'est pas une} affaire privée mais bien une nécessité pour la collectivité nationale.

charge. Mais c'est sans compter avec certains changements sociétaux; les traditions à elles seules ne suffisent plus, il faut ouvrir de nouvelles perspectives. Ce qui était autrefois une évidence, à savoir que les enfants reprennent le flambeau pour s'occuper de leurs parents qui ont tout fait pour leur progéniture mais ne sont plus en mesure de se prendre en charge eux-mêmes, est loin d'être acquis aujourd'hui. Précisément parce que la carrière professionnelle a énormément gagné en importance pour les femmes au cours des dernières décennies. Il est tout à fait possible que des enfants encore scolarisés et des grands-parents qui ont besoin d'être pris en charge doivent simultanément faire appel à la bonne grâce de la génération encore de plein pied dans la vie professionnelle. D'autant plus aujourd'hui que les mères assument leur rôle maternel plus tard que dans le passé. Alors que les femmes ont allié pendant de longues années le rôle de femme au foyer à celui de femme au travail, elles se voient aujourd'hui confrontées à une troisième mission: prendre soin de leurs aieuls. Situation qui soulève une pléthore de questions: les parents habitent-ils dans les environs ou très loin? Qui parmi les enfants serait le plus proche? Ai-je un frère ou une sœur qui pourrait être plus disponible que moi? Si oui (ou un autre parent proche), comment pourrions-nous nous partager les ment, est-ce possible et dans quelle limite? Qu'en est-il de notre budget? A qui puis-je me confier, parler de ma situation, de mon désespoir? Toute cette liste de questions n'est certainement pas exhaustive.

L'objectif est donc de dédier cette année aux problèmes et aux terrains de conflits mentionnés plus haut pour attirer l'attention de l'opinion. L'année 2011 placée sous le signe de l'«année du bénévolat» a permis d'aborder déjà certains points dans les médias, mais les aspects conflictuels n'en sont pas réglés pour autant. La tâche n'est pas simple non plus pour les médecins. D'un côté, il ne serait pas inutile d'avoir ces conflits présents à l'esprit dans notre quotidien professionnel, sans immédiatement se sentir obligé de venir en aide à tout le monde (un généraliste confiait dans un groupe de Balint: «Je ne peux pas faire en plus le boulot d'un travailleur social!»). D'un autre côté, c'est un appel lancé à tous les employeurs et/ou supérieurs hiérarchiques qui probablement réagissent souvent sans connaitre la situation dans laquelle se trouvent leurs employés, lorsque ceux-ci sont trop souvent absents, subissent une baisse de régime ou font valoir les prémisses d'un syndrome d'épuisement professionnel pour obtenir une réduction de leur temps de travail. Avec le risque de se voir attribuer de moins bonnes qualifications, voire de descendre dans la hiérarchie ou 


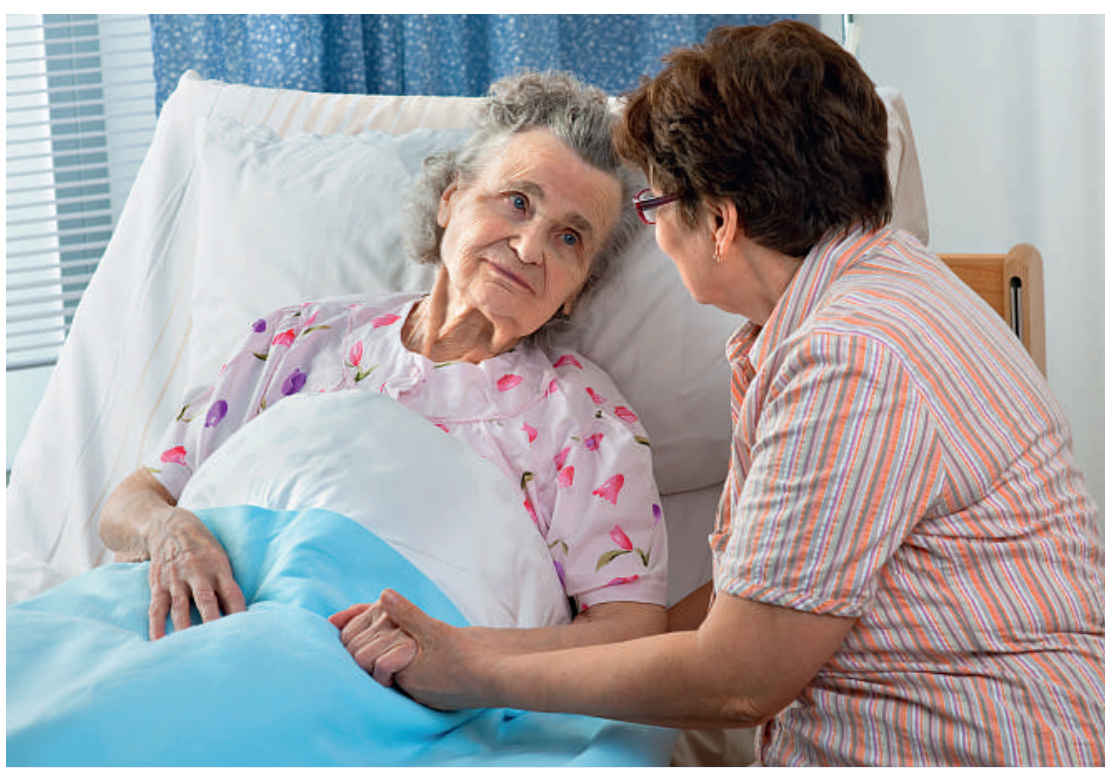

Alors que les femmes ont allié pendant de longues années le rôle de femme au foyer à celui de femme au travail, elles se voient aujourd'hui confrontées à une troisième mission: prendre soin de leurs aïeuls. pire, d'être licencié-e: autant de conséquences injustifiées pour tous ceux qui ont fait le choix de taire leur situation tout en essayant de rester à la hauteur de leurs performances habituelles. Des solutions viables pour l'avenir ne pourront voir le jour qu'à l'aune d'une approche partagée liant partenariat et perspectives. Dans une interview parue dans la Basler Zeitung du 7 janvier 2012, Stéphane Hessel, auteur français et ancien résistant, évoque le potentiel intarissable qui se cache dans chacun d'entre nous. Selon lui, nous devrions le faire fructifier pour développer de nouvelles approches: nous connaissons la prose mais il existe aussi la poésie (du grec poiêsis, "création»). Il s'agit donc de ne pas simplement suivre une ligne rationnelle en vue de légiférer mais de laisser de l'espace à la créativité. A l'instar de la neurobiologie qui nous enseigne que de nouvelles synapses neuronales ouvrent de nouvelles performances intellectuelles!

Selon diverses études, notre pays compte aujourd'hui quelque 200000 personnes [1] qui, tout en exerçant leur activité professionnelle, sont confrontées à la prise en charge d'un proche (aucune donnée exacte n'existe - et on connaît encore moins la situation des travailleurs indépendants), mais on sait par ailleurs que la majorité de ces personnes sont des femmes. Les hommes s'occupent la plupart du temps des tâches administratives de leurs aïeuls, ce qui signifie, en règle générale, un engagement moins prenant au quotidien qui se laisse plus facilement combiner avec une activité professionnelle.

En d'autres termes, il s'agit de questions extrêmement importantes qui concernent l'ensemble de notre société. Notre pays a mis en place des services d'aide et de soins à domicile qui cohabitent avec des services privés. Mais chez nous, on pratique également volontiers le recrutement de «migrantes pendulaires» (généralement des femmes des pays de l'Europe de l'Est qui, enrôlées par des agences spécialisées, font des allers et retours périodiques en alternance avec d'autres pour assurer la prise en charge d'un seul et même foyer; NZZ du 4 janvier 2012). Pour les personnes tributaires d'une aide et de soins extérieurs, mais souvent aussi pour les proches, ce genre de formule n'est pas satisfaisant; au contraire, c'est une contrainte supplémentaire. La politique de santé de notre pays souhaiterait que les personnes (très) âgées vivent le plus longtemps possible à la maison pour limiter la création de places coûteuses dans des centres de soins. Mais tout ce que cette vision réductrice ne veut pas voir existe malheureusement. Personne n'en tient compte et l'inavouable reste souvent caché. Toutefois, persister dans cette voie relèverait de l'hérésie. Il faut conjuguer les efforts des différentes parties pour arriver à ce que des solutions élaborées grâce à un réseau de partenariats puissent avoir la chance de voir le jour au niveau politique.

On écrit et on parle beaucoup - presque trop? de qualité de vie. Mais la plupart du temps, il est question de celle d'une seule personne ou d'un collectif déterminé de personnes. Pourtant, si effectivement seule la qualité de vie des patients est en ligne de mire - et c'est aussi ce que les médecins recherchent et ce que le public attend de nous - la qualité de vie de l'entourage du malade peut-elle être qu'une simple anecdote? Cela équivaudrait à pratiquer la politique de l'autruche et nous serions sanctionnés par de mauvaises notes. Mais nous ne serions pas les seuls: toutes les personnes actives dans les domaines de politique sanitaire et sociale sont elles aussi concernées. Une aubaine, car ainsi c'est tout un pan nouveau qui s'offre à nous, loin des seules considérations purement économiques. Cette question doit par ailleurs donner lieu à un large débat au sein de la population: des décisions politiques prises sans concertation seraient trop éloignées de la pratique. Finalement, on peut dire que l'humain dépend de la politique pour être au service de la société et que la politique ne serait rien sans l'humain lorsqu'elle planifie et légifère.

Pour de plus amples informations à ce sujet, veuillez consulter le site de la Journée des malades: www.tagderkranken.ch. Vous y trouverez des documents supplémentaires sur le thème de cette année.

\section{Référence}

1 Bischofberger I, Höglinger M. Herausforderungen für die Vereinbarkeit von Erwerbstätigkeit und Angehörigenpflege. Schweizer Arbeitgeber. 2008; 20:36-9. 